\title{
Diffeomorphic Registration Using B-Splines
}

\author{
Daniel Rueckert ${ }^{1}$, Paul Aljabar ${ }^{1}$, Rolf A. Heckemann ${ }^{2}$, Joseph V. Hajnal ${ }^{2}$, \\ and Alexander Hammers ${ }^{3}$ \\ ${ }^{1}$ Department of Computing, Imperial College London, UK \\ ${ }^{2}$ Imaging Sciences Department, MRC Clinical Sciences Centre, Imperial College London, UK \\ ${ }^{3}$ Division of Neuroscience and Mental Health, MRC Clinical Sciences Centre, \\ Imperial College London, UK
}

\begin{abstract}
In this paper we propose a diffeomorphic non-rigid registration algorithm based on free-form deformations (FFDs) which are modelled by B-splines. In contrast to existing non-rigid registration methods based on FFDs the proposed diffeomorphic non-rigid registration algorithm based on free-form deformations (FFDs) which are modelled by B-splines. To construct a diffeomorphic transformation we compose a sequence of free-form deformations while ensuring that individual FFDs are one-to-one transformations. We have evaluated the algorithm on 20 normal brain MR images which have been manually segmented into 67 anatomical structures. Using the agreement between manual segmentation and segmentation propagation as a measure of registration quality we have compared the algorithm to an existing FFD registration algorithm and a modified FFD registration algorithm which penalises non-diffeomorphic transformations. The results show that the proposed algorithm generates diffeomorphic transformations while providing similar levels of performance as the existing FFD registration algorithm in terms of registration accuracy.
\end{abstract}

\section{Introduction}

The area of computational anatomy is a rapidly developing discipline [1]. With the increasing resolution of anatomical scans of the human brain a number of computational approaches for characterising differences in the shape and neuro-anatomical configuration of different brains have emerged. Most morphometric techniques exploit the fact that mapping data sets into a standard space not only accounts for anatomic and functional variations and idiosyncrasies of each individual subject, but also offers a powerful framework which facilitates comparison of anatomy and function over time, between subjects, between groups of subjects and across sites. Non-rigid registration is a key element in many morphometric applications since it enables the warping of images into a standard reference space. Most non-rigid registration techniques use either elastic [2]3], fluid [4 5 6 67] or other deformation models [89|10 11/12].

In general, most registration algorithms make the assumption that similar structures are present in both images. Therefore it is desirable that the deformation field be smooth and invertible (so that every point in one image has a corresponding point in the other). Such smooth, invertible transformations are called diffeomorphisms. This is particularly important in cases where the output of the registration, i.e. the deformation field, is analyzed further, for example in deformation-based morphometry [1314]) or for the 
construction of statistical shape models [15]. However, in other applications diffeomorphisms are less important. For example, in registration-based segmentation the quality of the segmentation propagation is more important than the properties of the underlying deformation fields. Recent comparisons of non-rigid registration algorithms for segmentation propagation have shown that non-rigid registration techniques which do not guarantee diffeomorphisms can outperform those which guarantee diffeomorphic deformations [16].

There are a number of different ways of constructing diffeomorphic deformations. For example, in many fluid registration algorithms [45] the deformation process is stopped when the Jacobian of the transformation falls below a pre-defined threshold. The images are then regridded and the deformation process is allowed to continue. Other strategies include the composition of local warps which are diffeomorphic [15].

In this paper we propose a diffeomorphic non-rigid registration technique which uses a free-form deformation (FFD) model based on B-splines. To construct a diffeomorphic deformation we compose the deformation as a sequence of FFDs. By imposing hard constraints on the maximum deformation within each FFD we ensure that each individual FFD is a diffeomorphism. The composition of these FFDs is therefore also a diffeomorphism. We compare the proposed diffeomorphic registration algorithm to an existing free-form registration algorithm [8]. We also compare this to a soft-constraint approach in which topology preservation is enforced by introducing a penalty term which penalizes transformations for which the determinant of the Jacobian falls below a pre-defined threshold.

\section{Methods}

In practice, the anatomical variability between subjects cannot be sufficiently explained by an affine transformation which only accounts for differences due to position, orientation and size of the anatomy. To capture the anatomical variability, it is necessary to employ non-rigid transformations. A popular approach is to use spline-based transformations [8 17]. Using this approach, the deformation can be represented as a free-form deformation (FFD) based on B-splines which is a powerful tool for modelling 3D deformations and can be written as the 3D tensor product of the familiar 1D cubic B-splines,

$$
\mathbf{T}(\mathbf{x})=\sum_{l=0}^{3} \sum_{m=0}^{3} \sum_{n=0}^{3} B_{l}(u) B_{m}(v) B_{n}(w) \mathbf{c}_{i+l, j+m, k+n}
$$

where $\mathbf{c}$ denotes the control points which parameterise the transformation. The optimal transformation is found by minimising a cost function associated with the global transformation parameters as well as the local transformation parameters. The cost function comprises two competing goals: The first term represents the cost associated with the voxel-based similarity measure, in this case normalised mutual information [18], while the second term corresponds to a regularization term which constrains the transformation to be smooth [8]. The performance of this registration method is limited by the resolution of the control point mesh, which is linearly related to the computational complexity: more global and intrinsically smooth deformations can only be modelled using 
a coarse control point spacing, whereas more localized and intrinsically less smooth deformations require a finer spacing. To address this problem it is possible to use multilevel free-form deformations [19]8]. In this approach the free-form deformations at different control point spacings are added, i.e.:

$$
\mathbf{T}(x, y, z)=\sum_{i=1}^{n} \mathbf{T}_{i}(x, y, z)
$$

When using multi-level FFDs, the intrinsic regularization of B-splines is often sufficient to achieve smooth deformations.

\subsection{Diffeomorphic Non-rigid Registration Using Soft Constraints}

Recent studies have shown that this type of algorithm performs well in neuroimaging studies [16]. However, while this type of algorithm usually generates smooth deformations it does not guarantee that the resulting deformation field is diffeomorphic. One strategy to ensure that the FFD calculated by the non-rigid registration algorithm is diffeomorphic is to add a second term to the cost function that penalises any nondiffeomorphic transformations using the following penalty function $\mathcal{P}$ :

$$
\mathcal{P}= \begin{cases}\frac{\gamma^{2}}{\mid\left(\left.J(x, y, z)\right|^{2}\right.}-2 & \text { if }|J(x, y, z)| \leq \gamma \\ 0 & \text { otherwise }\end{cases}
$$

Here $|J(x, y, z)|$ represents the determinant of the Jacobian of the FFD. A similar penalty function has been first proposed by Edwards et al. [20] and effectively penalises any transformations for which the determinant of the Jacobian falls below a threshold $\gamma$. By penalising Jacobians that approach zero, we prevent the transformation from collapsing and keep it diffeomorphic. Note that simply using a smoothness penalty function would not be sufficient to guarantee a diffeomorphic transformation, since it is possible for a transformation to be smooth but non-diffeomorphic.

The cost function $\mathcal{C}$ which the registration algorithm minimizes is a combination of the image similarity $\mathcal{I}$ (normalised mutual information as before) and the penalty function $\mathcal{P}$ :

$$
\mathcal{C}=-\mathcal{I}+\lambda \mathcal{P}
$$

The weighting parameter $\lambda$ defines the trade-off between the image similarity and the penalty function. For the experiments in this paper $\gamma$ was set to 0.3 and $\lambda$ to 0.01 . This means that the penalty term is only activated if the determinant of the Jacobian drops below 0.3 .

\subsection{Diffeomorphic Non-rigid Registration Using Hard Constraints}

In the following we will use a strategy for ensuring diffeomorphic FFDs which is based on the work of Choi and Lee [21]. In their work the authors have derived sufficient conditions for injectivity which are represented in terms of control point displacements. These sufficient conditions can be easily tested and can be used to guarantee a diffeomorphic FFD. Without loss of generality we will assume that the control points are 
arranged on a lattice with unit spacing. Let $\Delta \mathbf{c}_{i, j, k}=\left(\Delta x_{i, j, k}, \Delta y_{i, j, k}, \Delta z_{i, j, k}\right)$ be the displacement of control point $\mathbf{c}_{i, j, k}$. Let $\delta_{x}=\max \left|\Delta x_{i, j, k}\right|, \delta_{y}=\max \left|\Delta y_{i, j, k}\right|$, $\delta_{z}=\max \left|\Delta z_{i, j, k}\right|$.

Theorem 1. A FFD based on cubic B-splines is locally injective over all the domain if $\delta_{x}<\frac{1}{K}, \delta_{y}<\frac{1}{K}$ and $\delta_{z}<\frac{1}{K}$.

Choi and Lee [21] have determined a value of $K \approx 2.48$ so that the maximum displacement of control points given by the bound $\frac{1}{K}$ is approximately 0.40 . This means that the maximum displacement of control points is given by the spacing of control points in the lattice. For example, for a lattice with $20 \mathrm{~mm}$ control point spacing the maximum control point displacement is $8 \mathrm{~mm}$ while for a lattice with $2.5 \mathrm{~mm}$ control point spacing the maximum control point displacement is $1 \mathrm{~mm}$.

In practice the bounds on the displacements are too small to model any realistic deformations. To model large deformations we use a composition of FFDs as proposed in [22]. For each FFD in this composition, the maximum control point displacement is limited by theorem 1 . This a fundamentally different to the multi-level FFDs mentioned earlier. In our approach the FFDs are concatenated, i.e.

$$
\mathbf{T}(x, y, z)=\mathbf{T}_{n} \circ \mathbf{T}_{n-1} \circ \cdots \circ \mathbf{T}_{2} \circ \mathbf{T}_{1}(x, y, z)
$$

so that the final deformation is a composition of FFDs. Since the composition of two diffeomorphisms produces a diffeomorphism we can construct a diffeomorphic deformation by ensuring that each individual FFD is diffeomorphic. Again, normalised mutual information is used as a similarity measure.

\section{Results}

\subsection{MRI Data and Manual Segmentations}

To evaluate the proposed registration algorithm we have tested the algorithms on 20 brain MR images from normal subjects. The data consisted of T1-weighted 3D volumes, acquired using an inversion recovery prepared fast spoiled gradient recall sequence (GE), TE/TR $4.2 \mathrm{msec}$ (fat and water in phase)/15.5 msec, time of inversion (TI) $450 \mathrm{msec}$, flip angle 20, to obtain 124 slices of $1.5 \mathrm{~mm}$ thickness with a field of view of $18 \times 24 \mathrm{~cm}$ with a $192 \times 256$ matrix, 1 NEX. Scanning took place at the National Society for Epilepsy MRI Unit (Chalfont St Peter, Buckinghamshire, UK). Scan data were resliced to create isotropic voxels of $0.9375 \times 0.9375 \times 0.9375 \mathrm{~mm}$, using windowed sinc interpolation.

Each data set was accompanied by a set of labels in the form of an annotated image volume, where every voxel was coded as one of 67 anatomical structures (or background, code 0 ). These labels had been prepared using a protocol for manually outlining anatomical structures on two-dimensional sections from the image volume. The protocol used was an augmented version of a published prototype [23].

\subsection{Evaluation of Non-rigid Registration Via Segmentation Comparison}

We have evaluated three registration techniques: the standard non-rigid registration as described in [8] (method A), a non-rigid registration based on the composition of FFDs 
described in section 2.2 (method B) and a non-rigid registration which penalizes nondiffeomorphic transformations described in section 2.1 (method C). For methods A and $C$ we used a coarse to fine approach with a control point spacing of $20 \mathrm{~mm}, 10 \mathrm{~mm}, 5 \mathrm{~mm}$ and $2.5 \mathrm{~mm}$. For method A we also used a coarse to fine approach with similar control point spacings. At each level of control point spacing up to 10 FFDs are concatenated so that the final transformation is a composition of up to 40 FFDs. We then chose randomly one of the 20 MR brain images as a reference image and all other 19 images were registered to the reference image. A visual example of the registrations is shown in Figure 1 In this example both method $\mathrm{A}$ and $\mathrm{B}$ lead to visually better alignment than method $\mathrm{C}$. A close-up of the corresponding deformation fields can be seen in Figure 2 The folding in the deformation field produced by method $\mathrm{A}$ is clearly visible while the deformation fields produced by the other two methods have no folding. However, it is also apparent that method B is better capable of modelling large deformations than method C.

The manual expert segmentations allow us to make an assessment of the relative performance of the registration algorithms in aligning specific structures or tissue classes. As a measure of agreement between the manual expert segmentation and those obtained via registration, we used the similarity index $(S I)$. For each anatomical structure $m$, it is defined as the ratio between the volume of the intersection (overlap) and the mean volume of the segmentations in the same coordinate space:

$$
S I_{m}=\frac{2 n\left(\mathcal{S}_{a}^{m} \cap \mathcal{S}_{b}^{m}\right)}{n\left(\mathcal{S}_{a}^{m}\right)+n\left(\mathcal{S}_{b}^{m}\right)}
$$

This measure ranges from 0 (for label sets that have no overlapping regions) to 1 (for label sets that are identical). Since the manual segmentation contains 67 anatomical structures, we have grouped the results into temporal, parietal, occipital and frontal cortex, grey matter nuclei, ventricular system, brain stem, cerrebellum and corpus callosum. The SIs reported for these groups of structures are the average SI values for the antomical structures within the groups. The results for all three algorithms are illustrated in Figure 3. For comparison we also include the SI values after affine registration only. Method A produces similar results to method B, and both are better than those obtained with Method C.

\subsection{Evaluation of the Deformation Fields Produced by Non-rigid Registration}

For all registration algorithms we have additionally computed the percentage of voxels within the brain for which the determinant of the Jacobian is negative. In the case of the standard non-rigid registration (method A) this percentage was $1.47 \%$ on average across all registrations. In contrast to this both other methods produced no voxels for which the determinant of the Jacobian was negative. For the non-rigid registration which penalizes non-diffeomorphic transformations (method $\mathrm{C}$ ) the trade-off between image similarity and penalty function was set to $\lambda=0.01$. In this case all voxels have a positive Jacobian determinant. To investigate the choice of $\lambda$ further we have repeated our experiments with values of $\lambda$ between $10^{-2}$ and $10^{-8}$. However, in these cases the percentage of voxels with negative Jacobian determinant is non-zero (increasing to $0.62 \%)$. This indicates that $\lambda$ of 0.01 is close to the optimal value ensuring no folding of the transformation. 


\section{Discussion and Conclusions}

In this paper we have proposed two registration methods for generating diffeomorphic FFDs. The first method is based on a penalty term which prevents the determinant of the Jacobian falling below a pre-defined threshold. The second method is based on a composition of FFDs. Each FFD within the composition is guaranteed to be diffeomorphic by limiting the maximum amount of control point displacement within an individual FFD. Our results have shown that the latter technique is able to achieve high registration accuracy (compared to exisiting FFD registration techniques) while generating diffeomorphic transformations. Our results have also shown that the first method generates diffeomorphic transformations, however at the expense of registration accuracy.

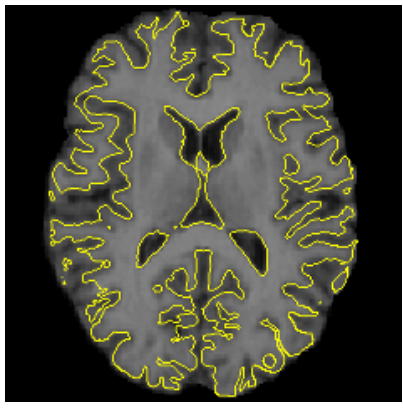

(a)

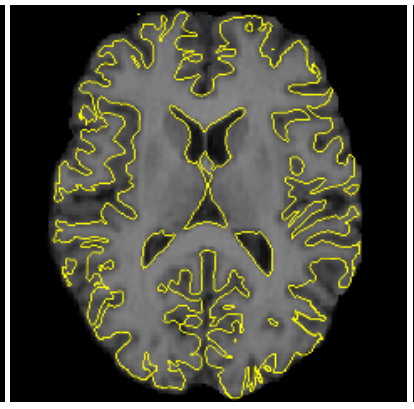

(b)

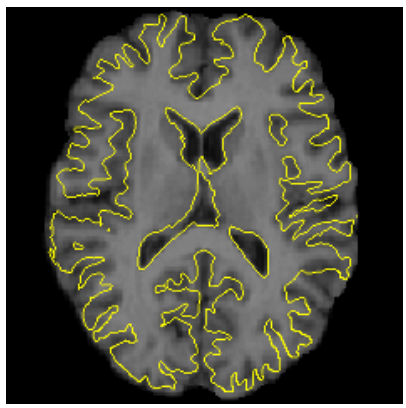

(c)

Fig. 1. This figure shows an example slice of a volumetric inter-subject registration: (a) after nonrigid registration using method $\mathrm{A}$, (b) after non-rigid registration using method $\mathrm{B}$ and (c) after non-rigid registration using method $\mathrm{C}$. The image shown is the reference image and the isolines shown are obtained from the transformed image.

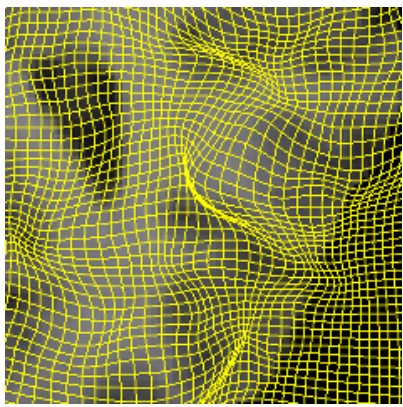

(a)

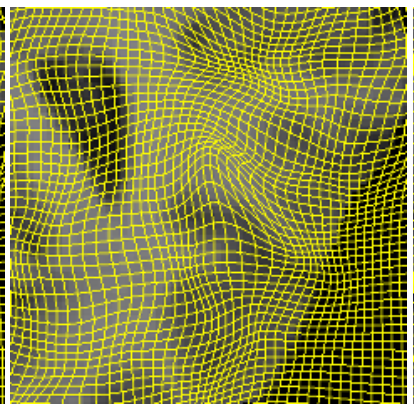

(b)

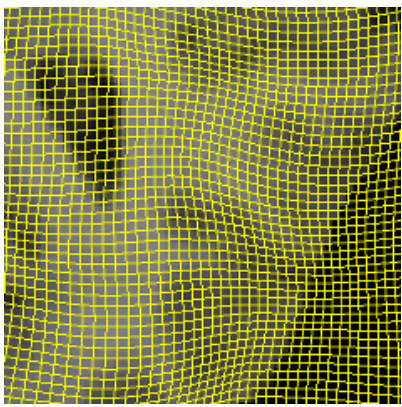

(c)

Fig. 2. This figure shows an example slice of the volumetric deformation produced by intersubject registration: (a) after non-rigid registration using method A, (b) after non-rigid registration using method B and (c) after non-rigid registration using method C. The folding is clearly visible in (a). There is no folding in (b) and (c), however (b) is better capable of modelling large deformations. 


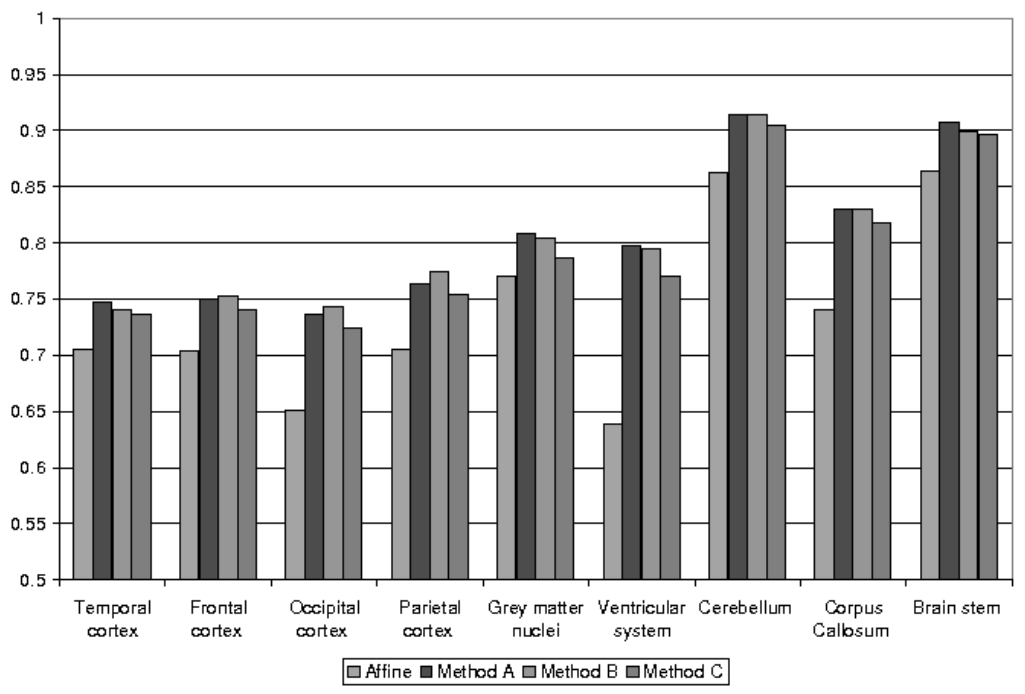

Fig. 3. This figure shows the SIs for different groups of anatomical structures

\section{References}

1. Grenander, U., Miller, M.I.: Computational anatomy: An emerging discipline. Quarterly of Applied Mathematics 56(4) (1998) 617-694

2. Bajcsy, R., Kovačič, S.: Multiresolution elastic matching. Computer Vision, Graphics and Image Processing 46 (1989) 1-21

3. Gee, J.C.: On matching brain volumes. Pattern Recognition 32(1) (1999) 99-111

4. Christensen, G.E., Rabbitt, R.D., Miller, M.I., Joshi, S.C., Grenander, U., Coogan, T.A., van Essen, D.C.: Topological properties of smooth anatomic maps. In: Information Processing in Medical Imaging: Proc. 14th International Conference (IPMI'95). (1995) 101-112

5. Christensen, G.E., Rabbitt, R.D., Miller, M.I.: Deformable templates using large deformation kinematics. IEEE Transactions on Image Processing 5(10) (1996) 1435-1447

6. Bro-Nielsen, M., Gramkow, C.: Fast fluid registration of medical images. In: Proc. 4th International Conference Visualization in Biomedical Computing (VBC'96). (1996) 267276

7. Beg, M.F., Miller, M.I., Trouvé, A., Younes, L.: Computing large deformation metric mappings via geodesic flows of diffeomorphisms. International Journal of Computer Vision 61(2) (2005) 139-157

8. Rueckert, D., Sonoda, L.I., Hayes, C., Hill, D.L.G., Leach, M.O., Hawkes, D.J.: Non-rigid registration using free-form deformations: Application to breast MR images. IEEE Transactions on Medical Imaging 18(8) (1999) 712-721

9. Davatzikos, C.: Spatial transformation and registration of brain images using elastically deformable models. Computer Vision and Image Understanding 66(2) (1997) 207-222

10. Hellier, P., Barillot, C., Mémin, É., Perex, P.: Hierarchical estimation of a dense deformation field for 3d robust registration. IEEE Transactions on Medical Imaging 20(5) (2001) 388-402

11. Shen, D., Davatzikos, C.: Hammer: Hierarchical attribute matching mechanism for elastic registration. IEEE Transactions on Medical Imaging 21(11) (2002) 1421-1439 
12. Thirion, J.P.: Image matching as a diffusion process: An analogy with Maxwell's demons. Medical Image Analysis 2(3) (1998) 243-260

13. Ashburner, J., Hutton, C., Frackowiak, R., Johnsrude, I., Price, C., Friston, K.: Identifying global anatomical differences: Deformation-based morphometry. Human Brain Mapping 6 (1998) 638-57

14. Chung, M.K., Worsley, K.J., Paus, T., C. Cherif, D.L.C., Giedd, J.N., Rapoport, J.L., Evans, A.C.: A unified statistical approach to deformation-based morphometry. NeuroImage 14(3) (2001) 595-606

15. Cootes, T.F., Marsland, S., Twining, C.J., Smith, K., Taylor, C.J.: Groupwise diffeomorphic non-rigid registration for automatic model building. In: ECCV. (2004) 316-327

16. Crum, W.R., Rueckert, D., Jenkinson, M., Kennedy, D., Smith, S.M.: A framework for detailed objective comparison of non-rigid registration algorithms in neuroimaging. In: Seventh Int. Conf. on Medical Image Computing and Computer-Assisted Intervention (MICCAI '04). (2004) 679-686

17. Kybic, J., Unser, M.: Fast parametric elastic image registration. IEEE Transactions on Image Processing 12(11) (2003) 1427-1442

18. Studholme, C., Hill, D.L.G., Hawkes, D.J.: An overlap invariant entropy measure of 3D medical image alignment. Pattern Recognition 32(1) (1998) 71-86

19. Lee, S., Wolberg, G., Chwa, K.Y., Shin, S.Y.: Image metamorphosis with scattered feature constraints. IEEE Transactions on Visualization and Computer Graphics 2(4) (1996) 337 354

20. Edwards, P.J., Hill, D.L.G., Little, J.A., Hawkes, D.J.: A three-component deformation model for image-guided surgery. Medical Image Analysis 2(4) (1998) 355-367

21. Choi, Y., Lee, S.: Injectivity conditions of 2D and 3D uniform cubic b-spline functions. Graphical Models 62(6) (2000) 411427

22. Hagenlocker, M., Fujimura, K.: CFFD: a tool for designing flexible shapes. The Visual Computer 14(5/6) (1998) 271-287

23. Hammers, A., Allom, R., Koepp, M.J., Free, S.L., Myers, R., Lemieux, L., Mitchell, T.N., Brooks, D.J., Duncan, J.S.: Three-dimensional maximum probability atlas of the human brain, with particular reference to the temporal lobe. Human Brain Mapping 19(4) (2003) 224-247 